\title{
Weber problems with alternative transportation systems
}

\author{
Emilio Carrizosa ${ }^{\text {a, * }}$, Antonio M. Rodríguez-Chía ${ }^{\text {b }}$ \\ a Facultad de Matemáticas, Universidad de Sevilla. C/Tarfia s/n, 41012 Sevilla, Spain \\ b Facultad de Ciencias del Mar, Universidad de Cádiz. Poligono Rio San Pedro, Puerto Real, Cádiz, Spain
}

Received I June 1995; accepted 1 December 1995

\begin{abstract}
In this paper we address a planar $p$-facility location problem where, together with a metric induced by a gauge, there exists a series of rapid transit lines, which can be used as alternative transportation system to reduce the total transportation cost. The location problem is reduced to solving a finite number of (multi)-Weber problems, from which localization results are obtained. In particular, it is shown that, if the gauge in use is polyhedral, then the problem is reduced to finding a $p$-median.
\end{abstract}

Keywords: Continuous location; Multi-Weber problems; Gauges; p-medians

\section{Introduction}

Most of the papers in the literature devoted to facility location consider the metric space to be the plane [8] - or the $n$-dimensional space - or a graph [10]. In this paper we present a mixed model, consisting of a $p$-facility minsum location problem on the plane, which is equipped with the metric obtained as the superposition of a planar metric (induced by a gauge) and a finite set of rapid transit lines. This enables us to model real-world situations in which, together with a homogeneous transportation system (e.g., a dense network of standard roads) there exists a series of rapid lines (e.g., highways), in which transit times are shorter.

An illustrative example, suggested by a referee,

\footnotetext{
* Corresponding author.
}

may be that of a pedestrian walking in a city with a subway network, to be used in order to reduce travel times.

To minimize the transportation costs, we assume, as in $[2,11]$ among others, that such costs are increasing concave functions of the distances involved, allowing even discontinuous functions such as fixed charge functions of the form

$f(t)= \begin{cases}0, & \text { if } t=0 \\ a+b t, & \text { if } t>0\end{cases}$

where $a, b \in \mathbb{R}^{+}$.

The paper is structured as follows. In Section 2 we address the calculus of shortest (minimum-cost) paths in the metric of the problem. In Section 3 we introduce the location problem, some localization properties of which are presented in Section 4, ending with an illustrative example. 
2. Alternative transportation systems. Minimumcost paths

\subsection{The basic model}

As mentioned in the Introduction, we consider that the space where the facilities are to be located is the plane $\mathbb{R}^{2}$, equipped with a metric derived from the mixture of the metric induced by a gauge $\|\cdot\|$ and the metric $d(\cdot, \cdot)$ of a complete directed metric graph $(N, E)$, whose node set $N$ is a finite subset of $\mathbb{R}^{2}$. Several proposals of mixtures of metrics can be found in [1,12] and references in [15].

We recall that the gauge $\|\cdot\|$ associated with the compact convex set $B$ with 0 at its interior is the function defined as

$\|x\|=\inf \left\{\mu>0: \frac{1}{\mu} x \in B\right\}$,

which is a norm iff $B$ is symmetric with respect to the origin.

Transportation costs are assumed to be given by nondecreasing concave functions of the distance travelled through the plane or through the rapid lines. In particular, let $g:[0,+\infty) \rightarrow \mathbb{R}$ and $\hat{g}:[0,+\infty) \rightarrow$ $\mathbb{R}$ be nondecrasing concave nonconstant functions such that

$g(\|y-x\|) \geq \hat{g}(d(x, y)) \quad \forall x, y \in N$,

$g(0)=\hat{g}(0)=0$,

and define the direct transportation cost from the point $x$ to the point $y$ as

$C(x, y)= \begin{cases}g(\|y-x\|), & \text { if either } x \text { or } y \notin N, \\ \hat{g}(d(x, y)), & \text { if } x, y \notin N .\end{cases}$

Condition (1) reflects the role of the graph in the space: its links give a set of rapid lines, such as highways, a railroad network, etc., along which travel is quicker (cheaper). This condition holds in practice as soon as the edges of the graph are truly rapid lines. Anyway, if, for some links, (1) does not hold, one can construct an equivalent graph verifying (1), see Section 2.2.

From the expression (3) for the cost of direct (i.e., without transhipment) transportation, the function $G$ that gives to any $(x, y) \in \mathbb{R}^{2} \times \mathbb{R}^{2}$ the minimum transportation cost from $x$ to $y$ is given by

$$
\begin{aligned}
G(x, y)= & \inf _{t_{1}, \ldots, t_{r}, r: \text { finite }} C\left(x, t_{1}\right)+C\left(t_{1}, t_{2}\right)+\ldots \\
& +C\left(t_{r-1}, t_{r}\right)+C\left(t_{r}, y\right) .
\end{aligned}
$$

The purpose of this section is to show that (4) can be simplified, by showing that the number of transhipment points in the optimal path from any $x$ to any $y$ is at most 2 .

Lemma 2.1. Let $h:[0,+\infty) \rightarrow \mathbb{B}$ be a concave function such that $h(0)=0$. One has

$h(x+y) \leq h(x)+h(y) \quad \forall x, y \geq 0$.

Proof. The case $x=y=0$ is trivial. For $x+y>0$, one has:

$$
\begin{aligned}
h(x) & =h\left(\frac{x}{x+y}(x+y)+\frac{y}{x+y} 0\right) \\
& \geq \frac{x}{x+y} h(x+y)+\frac{y}{x+y} h(0), \\
h(y) & =h\left(\frac{y}{x+y}(x+y)+\frac{x}{x+y} 0\right) \\
& \geq \frac{y}{x+y} h(x+y)+\frac{x}{x+y} h(0) .
\end{aligned}
$$

Adding both expressions, and setting $h(0)=0$, one obtains

$h(x)+h(y) \geq h(x+y)$,

as asserted.

Lemma 2.2. Let $M$ be a space equipped with the metric $m$. Let $h:[0,+\infty) \rightarrow \mathbb{R}^{+}$be a concave nondecreasing and nonconstant function $h \circ m$ is also a metric on $M$.

Proof. First of all, it is easy to show that

$h(t)>0 \quad \forall t>0$.

Hence, for any $x, y \in M, x \neq y$, since $m(x, y)>0$, $h(m(x, y))>0$. Moreover, $h(m(x, x))=h(0)=0$ for all $x \in M$. 
Now, we show the triangle inequality; by Lemma 2.1 and the triangle inequality for $m$,

$$
\begin{aligned}
& h(m(x, y))+h(m(y, z)) \\
& \quad \geq h(m(x, y)+m(y, z)) \geq h(m(x, z)) .
\end{aligned}
$$

Hence, $h \circ m$ is a metric on $M$.

Remark 2.1. As a consequence of Lemma 2.2, we see that the function $\hat{g} \circ\|\cdot\|$ is a metric on $N$ and the function $g \circ\|\cdot\|$ is a metric on $\mathbb{R}^{2}$ as soon as $\|\cdot\|$ is symmetric, i.e., a norm.

Proposition 2.1. For any $x \in \mathbb{R}^{2}, y \in N$, and $t_{1}, t_{2}, \ldots, t_{\mathrm{n}} \in \mathbb{R}^{2}$, there exists some $a \in N$ such that

$$
\begin{aligned}
& C\left(x, t_{1}\right)+C\left(t_{1}, t_{2}\right)+\ldots+C\left(t_{n}, y\right) \\
& \quad \geq C(x, a)+C(a, y) .
\end{aligned}
$$

As a consequence, the minimum transportation cost from $x \in \mathbb{R}^{2}$ to $y \in N$ is given by the minimum-cost path joining them and passing through at most one node of the graph, i.e.,

$G(x, y)=\min _{a \in N}\{C(x, a)+C(a, y)\}$.

Proof. We show that (5) holds for the different values of $n$. The result obviously holds for $n=0$ : take $a=y$. For $n=1$, there is some $t_{1} \in \mathbb{R}^{2}$ used as transhipment point. If $t_{1} \in N$, the result holds (take $a=t_{1}$ ); else, since $g$ is concave and nondecreasing and $\|\cdot\|$ is a gauge, we have that

$$
\begin{aligned}
C\left(x, t_{1}\right)+C\left(t_{1}, y\right) & =g\left(\left\|t_{1}-x\right\|\right)+g\left(\left\|y-t_{1}\right\|\right) \\
& \geq g\left(\left\|t_{1}-x\right\|+\left\|y-t_{1}\right\|\right) \\
& \geq g(\|y-x\|) \\
& \geq C(x, y),
\end{aligned}
$$

thus (5) holds taking $a=y$.

Let us now assume the result proved for any $x \in \mathbb{R}^{2}, y \in N, t_{1}, \ldots, t_{k} \in \mathbb{R}^{2}, k=0,1, \ldots, n$, and let us show that it also holds for $k=n+1$.

Given $t_{1}, \ldots, t_{n}, t_{n+1} \in \mathbb{R}^{2}$, since the result is assumed to be true for $k \leq n$, there exists $z^{1} \in N$ such that

$C\left(t_{1}, t_{2}\right)+\ldots+C\left(t_{n+1}, y\right) \geq C\left(t_{1}, z^{1}\right)+C\left(z^{1}, y\right)$.
On the other hand, we have shown (case $n=1$ ) that there exists $z^{2} \in N$ such that

$C\left(x, t_{1}\right)+C\left(t_{1}, z^{1}\right) \geq C\left(x, z^{2}\right)+C\left(z^{2}, z^{1}\right)$,

thus

$$
\begin{aligned}
& C\left(x, t_{1}\right)+C\left(t_{1}, t_{2}\right)+\ldots+C\left(t_{n+1}, y\right) \\
& \quad \geq C\left(x, t_{1}\right)+C\left(t_{1}, z^{1}\right)+C\left(z^{1}, y\right) \\
& \quad \geq C\left(x, z^{2}\right)+C\left(z^{2}, z^{1}\right)+C\left(z^{1}, y\right) \\
& \quad=C\left(x, z^{2}\right)+\hat{g}\left(d\left(z^{2}, z^{1}\right)\right)+\hat{g}\left(d\left(z^{1}, y\right)\right) \\
& \geq C\left(x, z^{2}\right)+\hat{g}\left(d\left(z^{2}, y\right)\right) \\
& \quad=C\left(x, z^{2}\right)+C\left(z^{2}, y\right),
\end{aligned}
$$

from which the result follows taking $a=z^{2} \in N$.

Corollary 2.1. For any $x, y \in \mathbb{R}^{2}$, there exist $a, b \in N$ such that

$$
\begin{gathered}
G(x, y) \\
=\min \left\{C(x, y), \min _{a, b \in N}\{C(x, a)+C(a, b)\right. \\
+C(b, y)\}\} \\
=\min \left\{g(\|y-x\|), \min _{a, b \in N} g(\|a-x\|)\right. \\
\quad+\hat{g}(d(a, b))+g(\|y-b\|)\} .
\end{gathered}
$$

Proof. We need to show that, given $r \leq 1$, $t_{1}, t_{2}, \ldots, t_{r} \in \mathbb{R}^{2}$, the cost $C\left(x, t_{1}\right)+C\left(t_{1}, t_{2}\right)$ $+\ldots+C\left(t_{r}, y\right)$ can be bounded below by an expression of the form $C(x, a)+C(a, b)+C(b, y)$ for some $a, b \in N$. Indeed, let $t_{r+1}=y$, and define the set $I$,

$I=\left\{k: 1 \leq k \leq r+1, t_{k} \in N\right\}$.

If $I$ is empty, we would have

$$
\begin{aligned}
& C\left(x, t_{1}\right)+C\left(t_{1}, t_{2}\right)+\ldots+C\left(t_{r}, y\right) \\
& \quad=g\left(\left\|t_{1}-x\right\|\right)+g\left(\left\|t_{2}-t_{1}\right\|\right)+\ldots+g\left(\left\|y-t_{r}\right\|\right) \\
& \quad \geq g\left(\left\|t_{1}-x\right\|+\left\|t_{2}-t_{1}\right\|+\ldots+\left\|y-t_{r}\right\|\right) \\
& \quad \geq g(\|y-x\|) \\
& \quad \geq C(x, y),
\end{aligned}
$$

and (6) follows. 
On the other hand, if $I \neq \varnothing$, denoting by $k$ the highest value in $I$, it follows from Proposition 2.1 that there exists some $a \in N$ such that

$C\left(x, t_{1}\right)+\ldots+C\left(t_{k-1}, t_{k}\right) \geq C(x, a)+C\left(a, t_{k}\right)$.

If $k=r+1$ (i.e., $t_{k}=y$ ), we are done; else, we have that

$\left\{t_{k+1}, t_{k+2}, \ldots, t_{r+1}\right\} \cap N=\varnothing$,

thus

$$
\begin{aligned}
& C\left(t_{k}, t_{k+1}\right)+\ldots+C\left(t_{r}, t_{r+1}\right) \\
& \quad=g\left(\left\|t_{k+1}-t_{k}\right\|\right)+\ldots+g\left(\left\|t_{r+1}-t_{r}\right\|\right) \\
& \quad \geq g\left(\left\|t_{r+1}-t_{k}\right\|\right) \\
& \quad=C\left(t_{k}, t_{r+1}\right) .
\end{aligned}
$$

Hence,

$$
\begin{aligned}
& C\left(x, t_{1}\right)+\ldots+C\left(t_{r}, t_{r+1}\right) \\
& \quad \geq C(x, a)+C\left(a, t_{k}\right)+C\left(t_{k}, t_{r+1}\right),
\end{aligned}
$$

with $a, t_{k} \in N$, and (6) follows.

\subsection{Extension}

The results of the previous subsection heavily rely on two facts: the graph $(N, E)$ is complete and $g \circ\|\cdot\|$ and $\hat{g} \circ d$ are related by expression (1).

In this subsection we show how to handle the situation in which either the graph is not complete or (1) does not hold.

We construct a metric $\hat{d}^{*}$ on $(N, N \times N)$ by performing the following steps:

Stcp 1. For each $x, y \in N$, we define $l(x, y)$ as

$l(x, y)= \begin{cases}+\infty, & \text { if }(x, y) \notin E, \\ \hat{g}(d(x, y)), & \text { if }(x, y) \in E .\end{cases}$

Step 2. For each $x, y \in N$, set

$l(x, y):=\min \{l(x, y), g(\|y-x\|)\}$.

Step 3. Perform a shortest-path algorithm for the digraph $(N, N \times N)$ with $l(x, y)$ as length of $(x, y)$.

\section{The multi-Weber problem}

\subsection{The model}

In this section we address a multi-Weber problem, (i.e., a $p$-facility minsum location which assigns each demand point to its closest facility $[15,16])$, under the metric considered in Section 2.1, which adds to the gauge a finite set of rapid transit lines, to be used when this represents a decrease in the total transportation cost.

Finding the set of $p$ points which minimize the total transportation cost amounts to solving the optimization problem

$$
\min _{x_{1}, \ldots, x_{p} \in \mathbb{R}^{2}} \sum_{i=1}^{n} \omega_{i} \min _{1 \leq j \leq p} G\left(x_{j}, a_{i}\right),
$$

where $D=\left\{a_{1}, \ldots, a_{n}\right\} \subset \mathbb{R}^{2}$ is the set of demand points, each $\omega_{i}>0$ represents the demand of $a_{i}$, $G(x, y)$ - given by (4) - represents the transportation cost of one unit of commodity from $x$ to $y$, and the variables $x_{1}, x_{2}, \ldots, x_{p}$ represent the coordinates of the $p$ facilities to be located.

By Proposition 2.1, the minimum-cost path from $x$ to each $a_{j}$ will visit at most two intermediate points of $N$. Hence, if we label as $n_{1}, n_{2}, \ldots, n_{m}$ the nodes of $N$, we can define the $0-1$ variables $s_{i j}$ and $t_{i j k l}$ as follows:

- $s_{i j}=1$ iff $a_{i}$ is served by facility $x_{j}$, following the direct path from $x_{j}$ to $a_{i}$, at a unit cost $g\left(\left\|a_{i}-x_{j}\right\|\right)$.

- $t_{i j k l}=1$ iff $a_{i}$ is served by $x_{j}$ following the path $x_{j} \rightarrow n_{k} \rightarrow n_{l} \rightarrow a_{i}$, at a unit cost $g\left(\left\|n_{k}-x_{j}\right\|\right)+$ $\hat{g}\left(d\left(n_{k}, n_{l}\right)\right)+g\left(\left\|a_{i}-n_{l}\right\|\right)$.

With these definitions, it is straightforward to check the following.

Proposition 3.1. Problem (8) is equivalent to

$$
\begin{array}{ll}
\min \quad \sum_{i=1}^{n} \sum_{j=1}^{p} \omega_{i}\left[\sum _ { k = 1 } ^ { m } \sum _ { l = 1 } ^ { m } t _ { i j k l } \left(C\left(x_{j}, n_{k}\right)\right.\right. & \left.+C\left(n_{k}, n_{l}\right)+C\left(n_{l}, a_{i}\right)\right) \\
& \left.+s_{i j} C\left(x_{j}, a_{i}\right)\right] \\
\text { s.t. } \quad & \sum_{j=1}^{p} s_{i j}+\sum_{k=1}^{m} \sum_{l=1}^{m} t_{i j k l}=1, \quad \forall i=1,2, \ldots n, \\
& s_{i j} \in\{0,1\} \quad \forall i=1,2, \ldots, n, \forall j=1,2, \ldots, p, \\
& t_{i j k l} \in\{0,1\} \quad \forall i=1,2, \ldots, n, \forall j=1,2, \ldots, p, \\
& \forall k, l=1,2, \ldots, m, \\
& x_{j} \in \mathbb{R}^{2} \quad \forall j=1,2, \ldots, p .
\end{array}
$$


When one fixes the binary variables $s_{i j}$ and $t_{i j k l}$ in (9), one obtains (7) a (multi)-Weber problem in which the role of demand points is played by the actual demand points $a_{1}, \ldots, a_{n}$ and by the nodes $n_{1}, \ldots, n_{m}$ as well. This implies the following.

Corollary 3.1. The resolution of Problem (8) can be reduced to solving a finite number of multi-Weber problems, whose set of demand points is a subset of $(D \cup N)$.

\section{Localization results}

Problem (8) is a nonconvex optimization problem, whose resolution is difficult due to the possible presence of local nonglobal optima. However, as shown in Corollary 3.1 , this problem is intimately related to the well-known Weber (case $p=1$ ) and multi-Weber problems, some properties of which are translated in this section into properties for (8). In particular, it is derived that, for some especial cases, the resolution of (8) can be reduced to the inspection of a finite set of points, thus converting the global optimization problem into a combinatorial one.

In order to give the localization results, some definitions are given. We refer the reader to $[3,7,9]$ for further details.

Definition 4.1. Let $\|\cdot\|$ be the gauge in $\mathbb{R}^{2}$ with unit ball $B$. Given $\left\{u_{a}\right\}_{a \in A} \subset B^{o}$, (the polar to $\mathrm{B}$ ), let $N_{B^{\prime \prime}}\left(u_{a}\right)$ be the normal cone of $B^{o}$ at $u_{a}$, i.e.,

$N_{B^{\prime}}\left(u_{a}\right)=\left\{x \in \mathbb{R}^{2} /\left\langle x, p-u_{a}\right\rangle \leq 0 \quad \forall p \in B^{o}\right\}$.

The set

$C\left(\left(u_{a}\right)_{a \in A}\right)=\bigcap_{a \in A}\left(a+N_{B^{\circ}}\left(u_{a}\right)\right)$

is called elementary convex set associated with $\left(u_{a}\right)_{a \in A}$.

Definition 4.2. The point $x^{*} \in \mathbb{R}^{2}$ is said to be an intersection point for $A$ if $x^{*}$ is an extreme point of some elementary convex set $C\left(\left(u_{a}\right)_{a \in A}\right)$.

This concept is particularly useful when $\|\cdot\|$ is a polyhedral norm (or gauge, in the asymmetric case), i.e., a norm (gauge) whose unit ball is a polytope in $\mathbb{R}^{2}[3,7,15,17]$, since in such a case the set of intersection points is a finite and easily constructed set. For instance, if $\|\cdot\|$ is the $l_{1}$ norm, then, the set of intersection points for $A$ is the set of nodes of the grid through the points of $A$ with lines parallel to the coordinate axes. See also Section 5.

Proposition 4.1. There exist $x_{1}^{*}, \ldots, x_{p}^{*}$, intersection points for $N \cup D$, which solve Problem (8).

Proof. In [7] it is shown that the set of intersection points contains an optimal solution to each (multi)Weber problem. The result then follows from Corollary 3.1 .

In particular, if $\|\cdot\|$ is a polyhedral gauge then Problem (8) is reduced to evaluating its objective function at the of intersection points, which is a finite set:

Corollary 4.1. Let $\|\cdot\|$ be a polyhedral gauge, and let $V=\left\{v_{1}, \ldots, v_{s}\right\}$ be the set of intersection points for $(D \cup N)$. Then, Problem (8) is equivalent to

$$
\begin{aligned}
& \min \sum_{i=1}^{n} \omega_{i} \min _{j} G\left(x_{j}, a_{i}\right) \\
& \text { s.t. } x_{1}, \ldots, x_{p} \in V .
\end{aligned}
$$

Remark 4.1. Problem (10) is a well studied problem in the literature of Locational Analysis, known as the p-median problem, for which a variety of very efficient exact algorithms (for small $p$ ) or heuristics has been proposed. See [10] for an excellent review.

In the non-polyhedral case, one may adapt the local-search procedures that have been designed for the (multi)-Weber problem, or, if the global optimum is sought, one may use techniques of Global Optimization [6], such as the Branch-and-Bound method known as BSSS [5,14].

However, it should be noted that Global Optimization techniques are very space- and time-consuming, thus only applicable in practice to small $p$ (say $p=1$ ). For higher values of $p$, one is forced to use heuristic methods.

The following result may be of help.

Proposition 4.2. If $\|\cdot\|$ is a norm, then, an optimal solution to Problem (8) lies at conv $(D \cup N)$, the convex hull of $D \cup N$. 
Table 1

Demand points, coordinates

\begin{tabular}{ll}
\hline$a_{1}$ & $(0,7)$ \\
$a_{2}$ & $(5,5)$ \\
$a_{3}$ & $(2,10)$ \\
$a_{4}$ & $(10,20)$ \\
$a_{5}$ & $(20,1)$ \\
$a_{6}$ & $(0,0)$ \\
$a_{7}$ & $(13,1)$ \\
\hline
\end{tabular}

Proof. It is shown e.g. in [4] that the convex hull contains an optimal solution to each (multi-) Weber problem. The result then follows from Corollary 3.1.

Hence, as soon as the gauge $\|\cdot\|$ is symmetric (i.e., $\|\cdot\|$ is a norm), and one is willing to solve the Problem (8) by the BSSS one can take conv $(D \cup N)$ as starting set. For the asymmetric case, set-bounds for the multi-Weber problem, such as those given in [13], can be easily adapted.

\section{An example}

Just for illustrative purposes, we present and solve a small example.

We assume that the set of demand points consists of 7 points, $D=\left\{a_{1}, a_{2}, \ldots, a_{7}\right\}$, all with the same demand $\omega$, and coordinates given in Table 1 .

The metric $\|\cdot\|$ over $\mathbb{R}^{2}$ is assumed to be the $l_{1}$ norm,

$\left\|\left(x_{1}, x_{2}\right)\right\|=\left|x_{1}\right|+\left|x_{2}\right| \quad \forall x=\left(x_{1}, x_{2}\right) \in \mathbb{R}^{2}$.

We also have imbedded in $\mathbb{R}^{2}$ the complete undirected graph $(N, E)$, with

$$
\begin{aligned}
N & =\left\{n_{1}, n_{2}, n_{3}\right\} \\
& =\{(0,0),(10,10),(13,1)\},
\end{aligned}
$$

equipped with the metric $d$,

$d(x, y)=\frac{1}{4}\|y-x\|$,

implying that travel speed along the edges of the graph is four times the speed through the plane, a situation that may appear in urban contexts, where, together with an extremely dense system of links (streets), one has a few high-capacity avenues or ring roads, along which travel times are lower.
Finally, we also assume that the transportations costs are increasing functions of the travel times, given by

$g(t)=\hat{g}(t)= \begin{cases}3+2 t, & \text { if } t>0, \\ 0, & \text { if } t=0 .\end{cases}$

Hence, the transportation cost $C(x, y)$ from $x$ to $y$ is given by

$$
C(x, y)=\left\{\begin{array}{l}
3+2\|y-x\|, \\
\quad \text { if either } x \notin N \text { or } y \notin N, x \neq y, \\
3+\frac{1}{2}\|y-x\|, \\
\quad \text { if } x, y \in N, x \neq y, \\
0, \quad \text { if } x=y .
\end{array}\right.
$$

The aim is to find the location for two facilities minimizing the total transportation cost from the demand points to their closest facility.

By Proposition 4.1, there exist $x_{1}^{*}, x_{2}^{*}$ which solve Problem (8) and belong to the set of intersection points generated by $A \cup N$.

Since the metric in use is the $l_{1}$ norm, the intersection points are the nodes of the grid depicted in Fig. 1.

Hence, an optimal solution to Problem (8) can be obtained by evaluating the objective function (using Corollary 6) at the $\left(\frac{36}{26}\right)$ possible pairs.

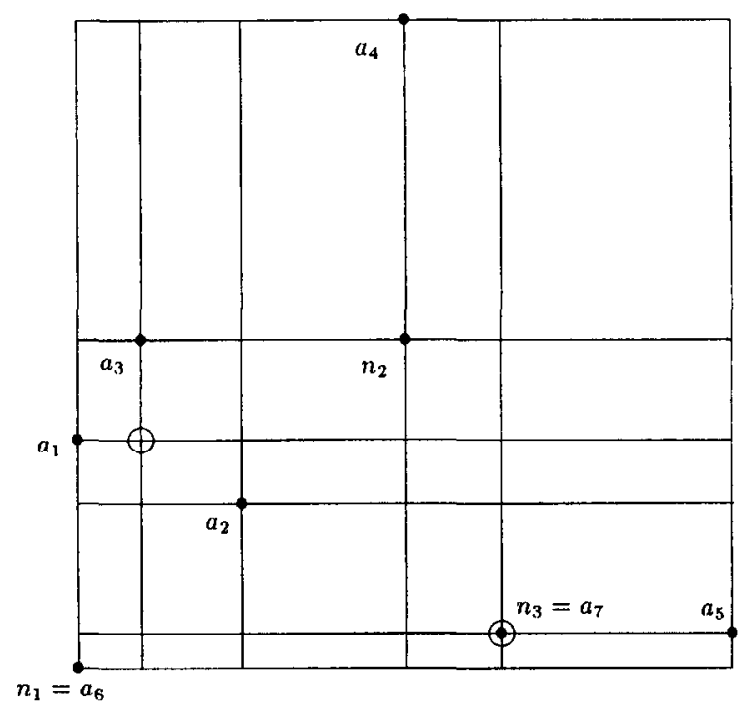

Fig. 1. Intersection points. 
Table 2

Allocations and routes

\begin{tabular}{lll}
\hline Demand point & Facility & Route \\
\hline$a_{1}$ & $x_{1}^{*}$ & $a_{1} \rightarrow x_{1}^{*}$ \\
$a_{2}$ & $x_{1}^{*}$ & $a_{2} \rightarrow x_{1}^{*}$ \\
$a_{3}$ & $x_{1}^{*}$ & $a_{3} \rightarrow x_{1}^{*}$ \\
$a_{4}$ & $x_{2}^{*}$ & $a_{4} \rightarrow n_{2} \rightarrow x_{2}^{*}$ \\
$a_{5}$ & $x_{2}^{*}$ & $a_{5} \rightarrow x_{2}^{*}$ \\
$a_{6}=n_{1}$ & $x_{2}^{*}$ & $n_{1} \rightarrow x_{2}^{*}$ \\
$a_{7}=n_{3}$ & $x_{2}^{*}$ & \\
\hline
\end{tabular}

\section{Such evaluation gives}

$x_{1}^{*}=(2,7), x_{2}^{*}=(13,1)$

as optimal, which are represented in Fig. 1 as circled points.

The allocation of demand points to facilities, as well as the routes used are given in Table 2 .

\section{Acknowledgements}

The research of the first author is partially supported by grant PB93-0927 of the Spanish DGICYT. This support is gratefully acknowledged.

\section{References}

[1] Batta, R., and Palekar, U.S., " Mixed planar/network facility location problems", COR 15 (1988) 61-67.

[2] Cooper, L., "Solutions of generalized locational equilibrium models", Journal of Regional Science 7 (1967) 1-18.

[3] Durier, R., and Michelot, C., "Geometrical properties of Fermat-Weber problem", European Journal of Operational Research 20 (1985) 332-343.
[4] Durier, R., and Michelot, C., "Sets of efficient points in a normed space', Journal of Mathematical Analysis and Applications 117 (1986) 506-528.

[5] Hansen, P., Peeters, D.. Richard, D., and Thisse. J.F.. "The minisum and minimax problems revisited", Operations $R e$ search 33 (1985) 1251-1265.

[6] Horst, R., and Tuy, H., Global Optimization, Deterministic Approaches, Springer-Verlag, 1990.

[7] Idrissi, H.F., Loridan, P., and Michelot, C., "Approximation of solutions for location problems", Journal of Optimization Theory and Applications 56 (1988) 127-143.

[8] Love, R.F., Morris, J.G., and Wesolowsky, G.O., Facilities Location: Models and Methods, North-Holland, Amsterdam, 1988.

[9] Michelot, C.. "The mathematics of continuous location", Studies in Locational Analysis 5 (1993) 59-83.

[10] Mirchandani, P.B., "The p-median problem and generalizations", in: P.B. Mirchandani and R.L. Francis (eds.), Discrete Location Theory, Wiley, 1990, 56-117.

[11] Morris, J.G., "Convergence of the Weiszfeld algorithm for Weber problems using a generalized 'distance' function", Operations Research 29 (1981) 37-48.

[12] Odoni, A., and Sadiq, G., "Two planar facility location problems with high-speed corridors and continuous demand", Working Paper, Massachusetts Institute of 'Technology, 1982.

[13] Plastria, F., "Localization in single facility location", European Journal of Operational Research 18 (1984) 215-219.

[14] Plastria, F., "The generalized big square small square method for planar single facility location", European Journal of Operational Research 62 (1992) 163-174.

[15] Plastria, F., "Continuous location problems: Research, results and questions", in: Z. Drezner (ed.), Facility Location: A Surtey of Applications and Methods. Springer-Verlag, 1995, 85-127

[16] Rosing, K., "An optimal method for solving the (generalized) nulti-Weber problem", European Journal of Operational Research 58 (1992) 414-426.

[17] Ward, J.E., and Wendell, R.E., "Using block norms for location modelling', Operations Research 33 (1985) 10741090 . 